\title{
HST/FOS SPECTRAL MAPPING OF IP PEGASI IN OUTBURST
}

\author{
R. K. Saito, ${ }^{1}$ R. Baptista, ${ }^{1}$ and K. Horne ${ }^{2}$
}

We report the results of a time-resolved eclipse mapping of the dwarf nova IP Pegasi during the decline of its May 1993 outburst from HST/FOS fast spectroscopy covering 3 eclipses in the spectral range $1100-2500 \AA$.

\section{INTRODUCTION}

IP Pegasi is a eclipsing dwarf nova $\left(P_{\text {orb }}=3.8 \mathrm{hr}\right)$ which shows $1-2$ weeks-long, $\simeq 2$ mag outbursts every 60-120 days. During outbursts, tidally induced spiral shocks form in its accretion disc as the disc expands and its outer parts feel more effectively the gravitational attraction of the companion star (Steeghs, Harlaftis \& Horne 1997). Here we report the results of a tirie-resolved ultraviolet (UV) spectral mapping experiment of IP Pegasi 9-13 days after onset of the May 1993 outburst.

\section{DATA ANALYSIS AND RESULTS}

Time-resolved spectroscopy covering 3 eclipses of IP Peg was secured with the Faint Object Spectrograph onboard the Hubble Space Telescope on 1993 May 27-30. The runs cover the eclipse cycles 22249 (IP5), 22252 (IP6) and 22263 (IP7), according to the ephemeris of Wolf et al. (1993).

The out-of-eclipse UV spectra show prominent Ly $\alpha$, Si IV $\lambda 1400$, C IV $\lambda 1550$ and He II $\lambda 1640$ emission lines as well as broad absorption bands possibly due to Fe II. The spectra were divided into 58 narrow passbands and light curves were extracted for each one. Maximum-entropy eclipse mapping techniques were used to solve for a map of the disc brightness distribution and for the flux of an additional uneclipsed component in each band (Baptista \& Steiner 1993). The line maps are brighter at disc centre, whereas the continuum maps show a flat brightness distribution with no pronounced central source. The IP5 continuum maps show an asymmetric structure at a similar azimuth and radius as one of the spiral arms seen in Baptista, Harlaftis \& Steeghs (2000). The other spiral arm has possibly been lost because of the incomplete phase coverage of the light curves

\footnotetext{
${ }^{1}$ Departamento de Física, Universidade Federal de Santa Catarina, Trindade, 88040-900, Florianópolis, SC, Brazil (saito@astro.ufsc.br).

${ }^{2}$ School of Physics and Astronomy, University of St. Andrews, KY16 9SS, Scotland, UK.
}

of this data set. The time sequence of eclipse maps shows the progressive fading of both line and continuum emission as the outburst ends.

Spatially resolved disc spectra show strong Si IV, CIV and He II lines, which appear in emission everywhere in the disc. The spectrum of the bright spot differs from the disc spectrum in the outer regions $\left(0.3-0.6 R_{\mathrm{L} 1}\right)$. The IP7 data show significant emission along the gas stream. The FWHM of the CIV line is approximately constant with radius, in contrast to the expected $v \propto R^{-1 / 2}$ law for gas in Keplerian orbits. The radial run of the FWHM of He II and Si IV is also flatter than the Keplerian expectation. This suggests that these lines do not arise in the disc photosphere, but possibly in a disc chromosphere + wind.

Blackbodies and LTE H I emission spectra give poor fits to the slope of the flat UV continuum in the spatially-resolved spectra and lead to inconsistent and uncomfortably high temperatures. We fitted Kurucz's (1979) stellar atmosphere models to the continuum emission at each disc radius in order to estimate the radial run of the $T_{\text {eff }}$. The temperatures range from $20000 \mathrm{~K}$ in the inner disc to $9000 \mathrm{~K}$ in the outer disc regions. This is in contrast with the previous optical results (Baptista, Haswell \& Thomas 2002), which points to flat brightness temperature distributions with temperatures in the range $9000-5000 \mathrm{~K}$. The $T(R)$ distributions are also flatter than the $T \propto R^{-3 / 4}$ law of steady-state discs model. The derived temperatures at the end of the outburst are everywhere higher than the critical temperature above which the gas should remain in a steady, high-mass accretion regime (Warner 1995).

\section{REFERENCES}

Baptista R., Steiner J. E., 1993. A\&A, 277, 331

Baptista R., Harlaftis E. T., Steeghs D., 2000. MNR.AS. 314,727

Baptista R., Haswell C. A., Thomas G., 2002. MNRAS. 334,198

Kurucz R. L., 1979. ApJS, 40, 1

Steeghs D., Harlaftis E. T., Horne K., 1997. MNR.AS. $290, \mathrm{~L} 28$

Warner B., 1995. Cataclysmic Variable Stars, Cambridge University Press, Cambridge

Wolf S., Mantel K. H., Horne K., Barwig H., Shoembs R., Baernbantner O., 1993, A\&A, 273, 160 